$\xi=-$ 国

\title{
Numerical Method for Modeling the Turbofan Engine Tonal Fan BPF Noise Generation and Propagation Accounting the SAS Complex Acoustic Impedance
}

\author{
Sergey F. Timushev ${ }^{1}$, Vladimir N. Gavrilyuk ${ }^{2}$, Andrey A. Aksenov ${ }^{3}$, Sergey A. Kharchenko ${ }^{4}$ \\ ${ }^{1,2}$ Rocket Engines Department and Scientific Research Center of New Technologies \\ of the Moscow Aviation Institute (National Research University) 125993, Moscow, 4, Volokolamskoye Highway, Russia \\ ${ }^{3,4}$ TESIS Ltd. \\ 127083 Moscow, 18/706, Unnatov Sreet, Russia \\ *Corresponding author E-mail:irico.harmony@gmail.com
}

\begin{abstract}
Noise reduction issues in the developed world receive an increasing attention, which is reflected in a great tightening of the noise level requirements. For the practical solution of this problem authors propose a new high-performance numerical modeling method of threedimensional tonal noise acoustic field generated by the fan as a bladed machine. The method is based on solving a boundary value problem for Fourier transformed convective wave equation with complex variables in a Cartesian coordinate system in the arbitrary domain with impedance boundary conditions by finite volume method. The noise source is set in the form of sound power on the surface near the rotor that bounds the area of the vortex perturbations (pseudosound). Sound power is determined by acoustic-vortex method. There are shown examples of the method validation and application.
\end{abstract}

Keywords: acoustic-vortex method; blade passing frequency; complex acoustic impedance; pressure pulsations; SAS; tonal noise.

\section{Introduction}

Noise reduction, in order to minimize negative impact of noise on human activity in the open space, residential, office and industrial buildings, cultural and religious institutions, car interiors, trains, aircraft and even spacecraft cabin becomes the most urgent problem as the noise influences essentially on the human health and productivity. These issues in the developed world receive increasing attention, which is reflected in a significant tightening of the requirements for limiting the noise level in accordance with ISO standards $[1,2]$. Numerical modeling of the computer cooling fan acoustics becomes an engineering problem of current interest [3] as well as for train fans [4] and modern aircraft turbofan engines [5].

The nature of aerodynamic noise generation in the axial fan described in a very practical book of A. Guedel [6]. He noted that the fan noise spectrum comprises the tonal and broadband noise components. Tonal components at frequencies that are multiples of the rotor blade passing frequency (BPF) usually dominates in the spectrum and defines the total sound power level. In axial fans, where the circular velocity Mach number at the rotor periphery is below 0.5 , noise generation relates to the case of a dipole source following the aeroacoustic analogy introduced by M.J. Lighthill [7]. N. Curle [8], J.E. Flowcs Williams and D.L. Hawkings [9] formulated the theoretical basis for methods of the bladed machine aerodynamic noise computation. The dipole nature of noise generation and radiation is caused by forces acting on the rotor and stator blades from the gas flow pressure. The analytical formulation of these processes is based on the formalization [10] of W.R.
Sears and represents the radiated sound in the form of the socalled spinning modes [11]. J.M. Tyler and T.G. Sofrin [12] showed that spinning modes attenuated exponentially in a long pipe at low Mach flow velocity.

Currently computational fluid dynamics and acoustics methods widely developed that proposed to determine the fan acoustic radiation[13,14]. They are mainly based on the application of Lighthill equation and aeroacoustic analogy like FWH equation or the Kirchhoff theorem application [15]. In such methods the decomposition problem, i.e., separation of acoustic and vortex (pseudosound) modes in the area of the oscillation source is not resolved.

For the practical solution of the tonal noise problem authors propose a new high-performance numerical modeling method of three-dimensional acoustic field at blade passing frequencies, its higher and combined harmonics generated in bladed machines of computer devices, air conditioning systems, turbofan aircraft engines.

The method is based on solving a boundary value problem for Fourier transformed convective wave equation with complex variables in a Cartesian coordinate system in the arbitrary domain with impedance boundary conditions by the finite volume method. The noise source is set in the form of sound power on the surface near the rotor that bounds the area of the vortex perturbations (pseudosound). The sound power is determined by acoustic-vortex method [16]. In the acoustic-vortex method the noise is represented with a local derivative of the vortex mode pressure [17]. It identifies the source, pressure pulsations, and noise propagation in the near field as a direct result of numerical simulation. 


\section{Method}

\subsection{Governing Equation and Boundary Conditions}

The proposed numerical method of modeling 3-dimensional acoustic fields is based on solving boundary value problems in the field of arbitrarily complex geometry with respect to the Fourier image of the pressure perturbations $p$.

\section{Governing equation}

Governing equation describes the propagation of sound in a thermodynamically uniform

$$
\left(i k+M_{j} \frac{\partial}{\partial x_{j}}\right)^{2} p=\frac{\partial^{2} p}{\partial x_{j}^{2}}+f
$$

steady flow. Flow parameters varies in the space with the Mach number vector $M_{j}$ [18]. Here $k=\omega / c$, where $\omega$ - frequency $c$ - sound speed.

\section{Boundary conditions}

The boundary condition at the wall can be written in the form of Myers relation [19] as:

$i k u_{n}=\left(i k+M_{j} \frac{\partial}{\partial x_{j}}-\frac{\partial M_{l}}{\partial x_{j}} n_{j} n_{l}\right) \frac{p}{\rho c Z}$,

where $u_{n}=n_{j} u_{j}$ - the Fourier image of the velocity perturbation directed to the normal to the wall $n_{i}, Z$ - specific complex impedance of wall or sound-absorbing structure (SAS), in general, dependent on the local Mach number and the boundary layer parameters.

On the perfectly reflecting wall boundary condition (2) becomes $u_{n}=0$. On the external boundaries of the computational domain the non-reflective boundary condition is specified as a Sommerfeld sound emission into unlimited space, here modified to take into account space non-uniformity of sound wave parameters in accordance with the concept [20] :

$i k \rho c u_{n}=\left(i k+M_{j} \frac{\partial}{\partial x_{j}}-\frac{\partial M_{l}}{\partial x_{j}} n_{j} n_{l}\right) p+\frac{i}{2 k} \Delta_{n} p$,

where $\Delta$ - two-dimensional Laplace operator in the plane, orthogonal to the normal vector $n_{j}$.

To close the boundary conditions $(2,3)$ the Fourier transform of momentum conservation law projection on the normal is used, that can be written as follow:

$$
\left(i k n_{j}+\frac{\partial M_{l}}{\partial x_{j}} n_{l}\right) u_{j}+\left(M_{l} \frac{\partial u_{j}}{\partial x_{l}}+\frac{1}{\rho c} \frac{\partial p}{\partial x_{j}}\right) n_{j}=0
$$

\subsection{Acoustic Source}

The acoustic source is modeled in the form of boundary conditions at ambient fan control surface $\mathrm{S}$ remote from its rotor by a distance at which attenuation of vortex or pseudo-sound mode is almost complete and the oscillations are primarily acoustic (usually this distance is about 1.5 of the chord length of rotor blades
$[18,21]$. This boundary condition can be written in the form of a right invariant

$J_{+}(x)=p+\rho c u_{j} n_{j}$,

in which the pressure and velocity perturbations are determined by either:

From a single 3-dimensional calculation of unsteady turbulent flow in the fan taking into account the rotor-stator interaction by moving meshes technique [22];

Simple iterations of the energy equation that can be written in the integral form [16,21] (6) with known sound source power defined by acoustic-vortex method and

- $\quad$ sound wavelength considerably larger the rotor radius $R / \lambda<<1$;

- Mach number in the region bounded by the $\mathrm{S}$ is much less than $1-M<<1$.

$\frac{1}{\sqrt{2 \pi}} \int_{S}^{+\infty} \int_{-\infty}^{+\infty} p(\omega-\xi) u_{n}(\xi) d \xi \cdot d S=W_{l} \delta\left(\omega-\omega_{l}\right)$

where $W_{l}$ - sound source power at the BPF harmonic $l$.

\subsection{Computational Grid}

The problem (1-4) is solved by the finite volume method on Cartesian grid, agreed with the boundary of computational domain automatically fitted to the body shape and features of the solution through the h-refinement procedure. Fig. 1 shows a fragment of a body immersed in a Cartesian grid.

Fig. 2 outlines the behavior of the integral L1 norm of local truncation error for model scalar transfer equation in an annular region for structured and unstructured triangular and curvilinear sequence refined grids [23].

Ошибка! Объект не может быть создан из кодов полей редактирования.

Fig. 1: Cartesian immersed-body grid

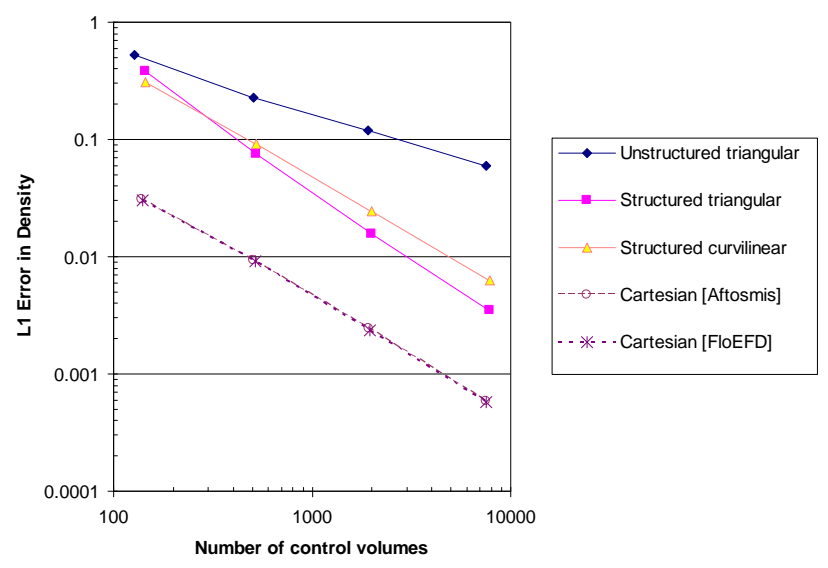

Fig. 2: L1 norm of local truncation error for sequence of refined meshes. The lower (dashed) curves were obtained on a Cartesian grid

It can be seen that the smallest error of approximation is reached on a Cartesian grid.

Besides Cartesian grids enables the integration of computational technology developed in the CAD system [24]

\subsection{Parallel Iterative Algorithm for SLAE Solver}

The parallel iterative algorithm for solving the system of linear algebraic equations (SLAE) derived as a result of discretization of equations $(1-4)$. The initial SLAE is given in complex numbers. 
In order to use the SLAE solver for real numbers the initial SLAE is transformed into the equivalent real form as a SLAE with double sized dimension. Each pair of real unknowns that form one complex number is treated as a whole block of unknowns, that is equivalent to the complex SLAE as the real block sparse SLAE with small $2 \times 2$ dense blocks. The parallel FlowVision solver TParFBSS $[25,26]$ is applied for solving the SLAE. The iterative algorithm for solving SLAE in TParFBSS solver consists of several stages. At the first stage Nested Dissection type ordering based on $2 \times 2$ block sparsity is computed. This ordering is further used to specify the parallel structure of computations. At the second stage in parallel to the tree structure levels the high order incomplete block triangular factorization of the SLAE matrix is computed. Finally at the third stage the SLAE solution is computed by performing some number of iterations of preconditioned Krylov type algorithm. Heterogeneous parallel implementation of the whole iterative solution algorithm is performed using MPI standard over the distributed memory and using Intel TBB technology over the shared memory.

\section{Validation Examples}

\subsection{Fan in the Pipe}

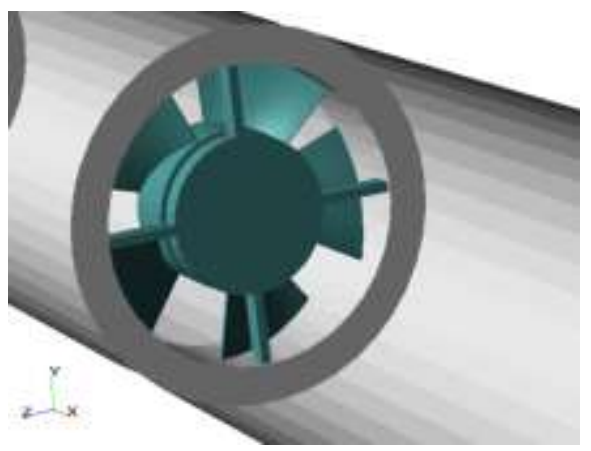

(a)

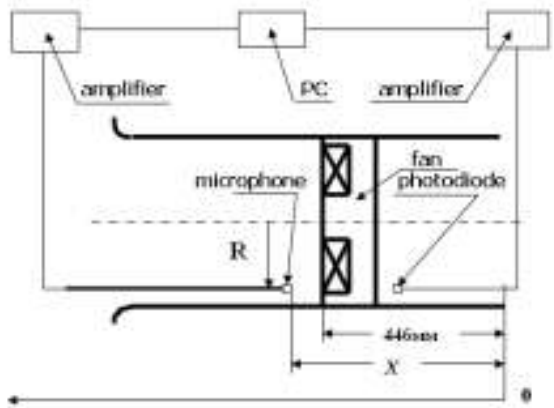

(b)

Fig. 3: Fan installation in the tube (a) and the sketch of pressure pulsation measurements (b)

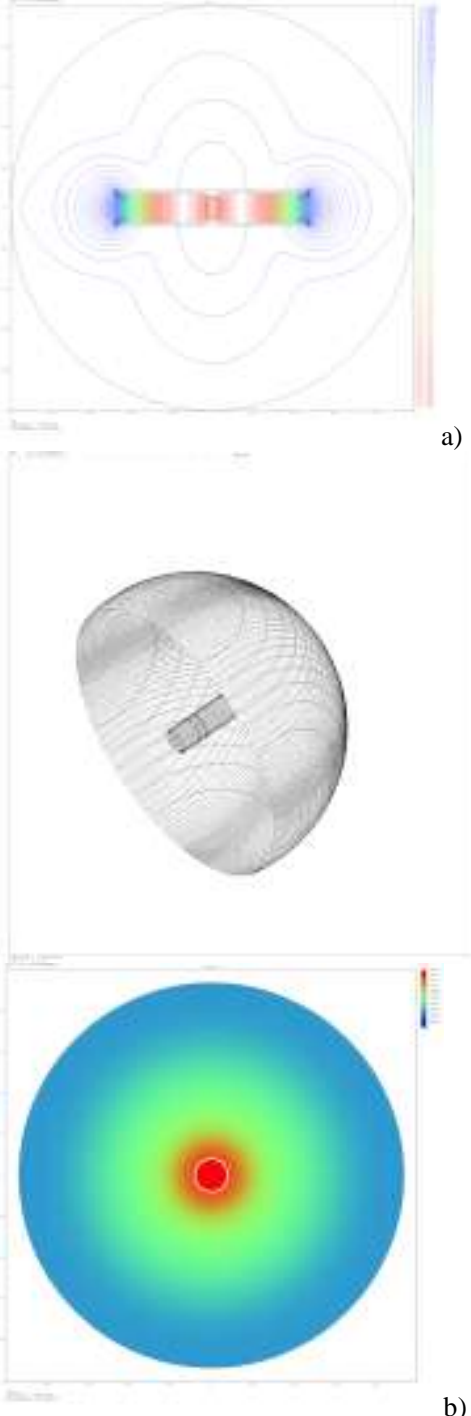

b)

Fig. 4: Mesh and pressure pulsations amplitude in the symmetry plane (a) and in a transverse plane (b)

Comparison the computation versus experiment for the calculation of tonal noise at the first BPF harmonic $222.5 \mathrm{~Hz}$ is considered. The tonal noise is generated by the fan VN-2 with a rotor of 60 $\mathrm{mm}$ radius. In the computational experiment the fan is installed in the pipe placed in a sphere with non-reflective boundary.

Computational grid in the symmetry plane and at the pipe and the non-reflecting wall of the spherical domain and pressure pulsations amplitude in the symmetry and transverse planes are shown in Fig. 4.

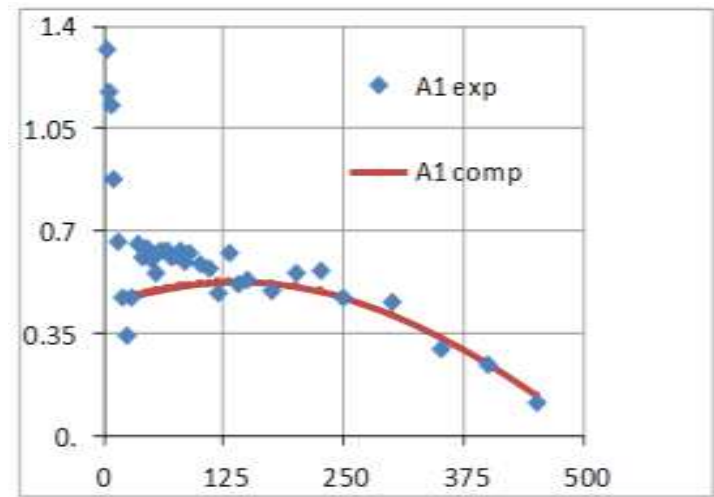

Fig. 5: Computed and experimental pressure pulsations amplitude $\mathrm{A} 1(\mathrm{~Pa})$ distribution along the length $(\mathrm{mm})$ of the pipe internal wall at radius $r=73$ $\mathrm{mm}$ upstream of the fan rotor 
Computed and experimental pressure pulsations amplitude distributions along the pipe length internal wall are shown in Fig. 5.

The amplitude of pressure pulsations upstream and downstream the rotor of radius $\mathrm{R}=60 \mathrm{~mm}$ drops sharply in pseudosound zone $(\mathrm{x}<1.5 \mathrm{R} \sim 100 \mathrm{~mm}$ ). Beyond this zone (where there are practically no pseudosound oscillations) the agreement between experimental and calculated data is quite satisfactory.

This task with 250,000 control volumes solved within $\sim 1$ minute on the 4 core CPU i7-3930K CPU $3.20 \mathrm{GHz}$.

\subsection{Plane Wave Reflection}

The plane sound wave in a space filled with moving from the right to the left (against x-direction) medium with averaged Mach number $M$ and limited from the left by plane $x=0$ on which the acoustic source specified by (5), and on the right by an acoustically perfect reflecting plane $x=L$ is considered.

The wave equation (1) in this case is simplified to the form:

$$
\left(1-M^{2}\right) \frac{\partial^{2} p}{\partial x^{2}}-2 i k M \frac{\partial p}{\partial x}+k^{2} p=0
$$

With the boundary conditions:

$$
\left(J_{+}\right)_{x=0}=(p+\rho c u)_{x=0},(u)_{x=L}=0
$$

The analytical solution of the problem $(7,8)$ can be represented as the sum of satisfying the equation (7) forward and backward waves:

$$
p=A e^{i k_{-} x}+B e^{-i k_{+} x}
$$

where $A, B$ - parameters, that can be found from boundary conditions (8):

$$
A=\frac{1}{i} \frac{1}{\left(k_{+}-k\right)-\frac{k_{+}}{k_{-}}\left(k_{-}+k\right) e^{i\left(k_{+}+k_{+}\right) L}}, \quad B=\frac{k_{+}}{k_{-}} e^{i\left(k_{+}+k_{+}\right) L} A
$$

where $k_{-}=k /(1-M), k_{+}=k /(1+M)$ are wave numbers accounting the medium motion Doppler effect.

In order to analyze the accuracy of the numerical solution of the problem $(7,8)$ let us consider the case:

$k=\pi=3.141592,\left(J_{+}\right)_{x=0}=(1,0), L=6$.

Numerical solutions for this case for $M=0$ and $M=1 / \sqrt{2}$ are shown in Fig. 6 as the amplitude of pressure pulsations $|p|$.

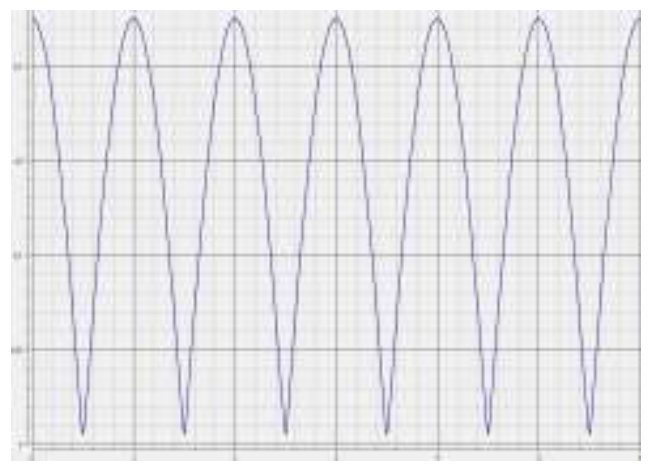

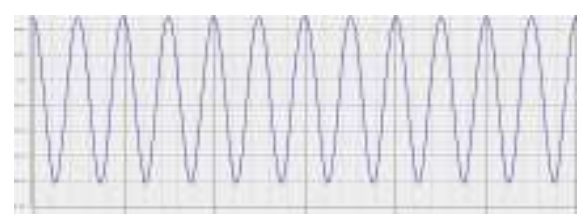

Fig. 6: Pressure $|p|$ as $x$ function. On the top - $M=0$, on the bottom $M=1 / \sqrt{2}$

A decrease in the period of the pressure spatial inhomogeneity in 2 times and increase of the minimum pressure module values are due to the Doppler effect for both forward and backward waves. Table 1 shows the influence of grid density on the accuracy of the numerical solution, described by the relative difference in the L1 norm between calculation and analytical solutions:

$$
E=\frac{\sum_{\text {cells }}\left|p_{\text {comp }}-p_{\text {theory }}\right|}{\sum_{\text {cells }}\left|p_{\text {theory }}\right|} \cdot 100 \%
$$

Table 1: Mesh convergence with E

\begin{tabular}{|l|l|l|}
\hline \multicolumn{1}{|l|}{$M=0$} & $\begin{array}{l}\text { Number of cells per 1/2 } \\
\text { period }\end{array}$ Cells & $E \%$ \\
\hline 100 & 8 & 0.2 \\
\hline 200 & 16 & 0.03 \\
\hline 400 & 32 & 0.004 \\
\hline
\end{tabular}

$M=1 / \sqrt{2}$

\begin{tabular}{|l|l|l|}
\hline Cells & $\begin{array}{l}\text { Number of cells per 1/2 } \\
\text { period }\end{array}$ & $E \%$ \\
\hline 100 & 4 & 13 \\
\hline 200 & 8 & 1.3 \\
\hline 400 & 16 & 0.13 \\
\hline
\end{tabular}

There is a very good convergence of the numerical solution to the exact one with increasing mesh density in both cases. However, the accuracy of solution for $M=1 / \sqrt{2}$ and the same mesh density significantly (about in $\sim 5$ times) lower than the accuracy of solution for $M=0$. It can be explained by the increasing of equation system complexity due to the convective term in wave equation proportional to the Mach number.

\subsection{Simple Silencer}

B. J. Tester suggested an expression for the optimal (so-called Cremer) impedance of the infinite circular duct specified at its internal wall. He also included a correction for mean flow effects:

$$
Z_{c r}=(0.88-0.38 i) \frac{k r}{\pi(1+M)^{2}}, \quad r \text { - duct radius }
$$

and presented appropriate theoretical maximum damping (TL) [27].

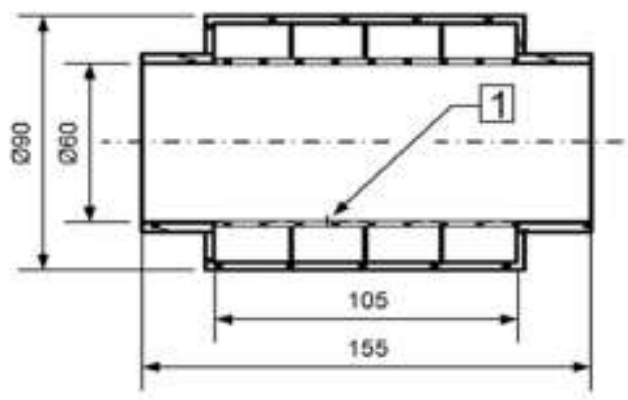

Fig. 7: Geometry of silencer studied. Impedance $Z_{C r}$ is specified at surface marked by 1 (length $105 \mathrm{~mm}$ ) 

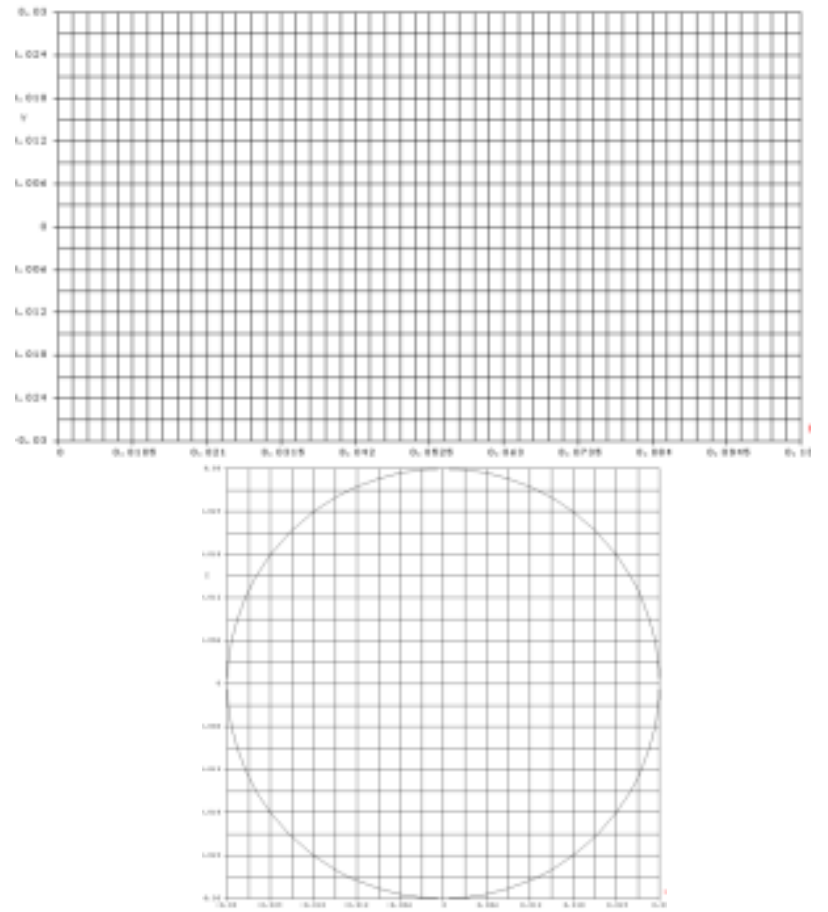

Fig. 8: Computational domain and mesh at the plane of symmetry and at the cross-section

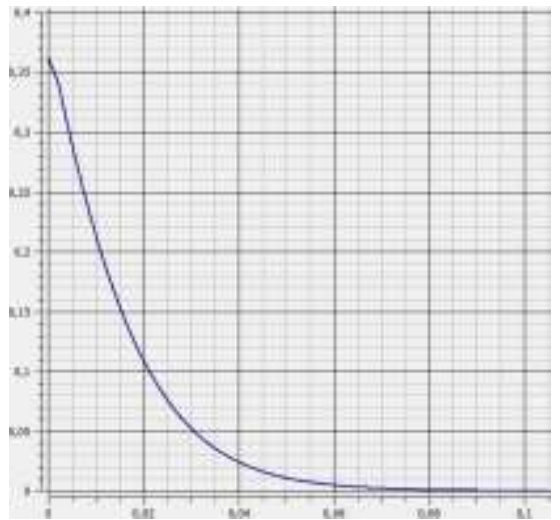

Fig. 9: Acoustic pressure amplitude distribution at the centerline

Below in Table 2 theoretical and computed results are presented.

Table 2: Sound transmission loss $(\mathrm{TL}=10 \operatorname{lgPin} / \mathrm{Pout})$ as a function of frequency

\begin{tabular}{|c|l|l|}
\hline $\mathrm{f} \mathrm{Hz}$ & TL, dB in Ref. [27] & TL, dB computed \\
\hline 350 & 81 & 81.1 \\
\hline 700 & 76 & 76.3 \\
\hline 1050 & 72 & 72.7 \\
\hline 1400 & 70 & 70.6 \\
\hline 1750 & 67.5 & 67.5 \\
\hline 2100 & 65 & 66.2 \\
\hline 2450 & 62.5 & 62.8 \\
\hline
\end{tabular}

One can see that 3-D computations are in a very well agreement with theory in [Error! Bookmark not defined.].

\section{Aircraft Engine Model Acoustics}

The stationary and disturbed flow in the aircraft engine model inlet part created by the incoming flow with a Mach number equal to 0.3 and the tonal noise at a frequency of $2100 \mathrm{~Hz}$ generated by the fan with 16 blades are considered. Model source of the fan disturbance is set analytically in the form of angle periodic with period 360/16 degrees and $1000 \mathrm{~Pa}$ amplitude function: $J_{+}=1000 \cdot[1+\cos (16 \phi)]$. The acoustic analysis is performed in a case of the presence at the air intake inner wall the SAS with a specific acoustic impedance $Z=4-0.5 i$ and in a case of SAS absence. The aim of the calculation is to determine the radiated sound power and sound radiation pattern diagram.

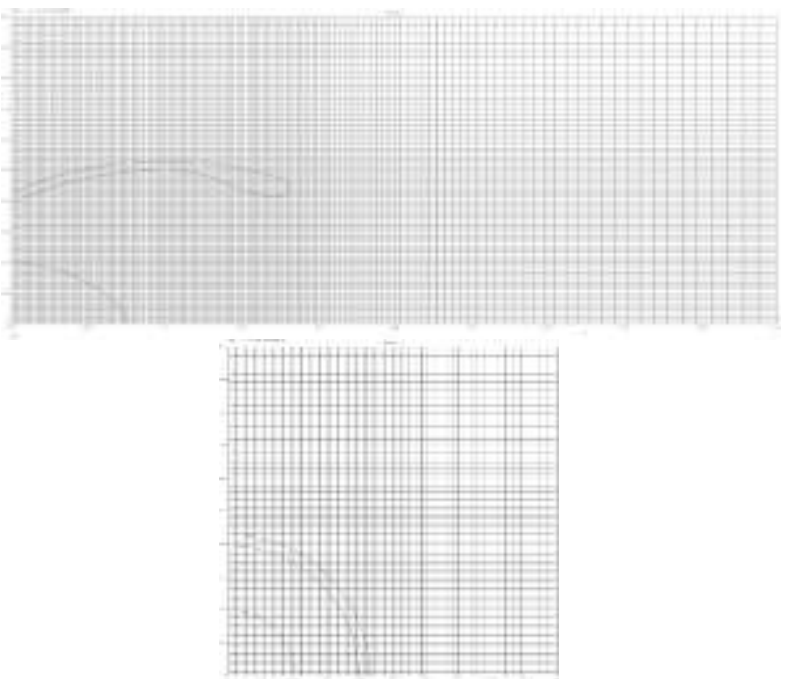

Fig. 10: Grid $(140 \times 72 \times 72)$ contains 709207 cells. Grid at the plain of symmetry (top) and in the plane in vicinity of fan (bottom)

Computational domain, grid and contours of the model are shown in Fig. 10. A sufficient mesh density inside the air intake ensures restriction of Helmholtz grid number $H<1$ according [28]. This mesh is used both for the stationary flow and acoustic computations.

Mach number field, included in the equation (1) and the boundary conditions (2-4), is obtained from preliminary computed the stationary axisymmetric turbulent flow. Mach number field in the symmetry plane is shown in Fig. 11. There is shown a significant heterogeneity of this field.

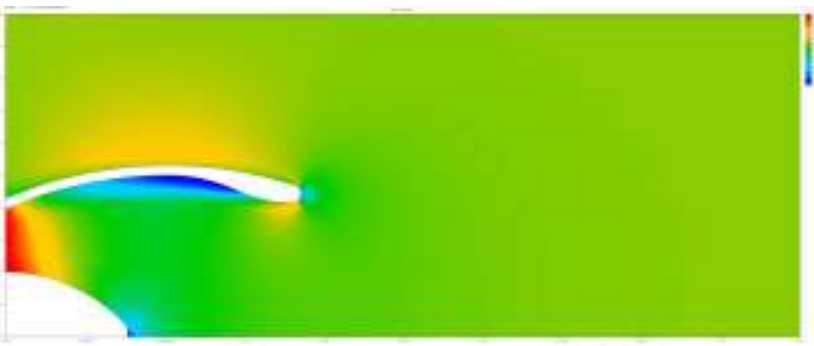

Fig. 11: Mach number in the plane of symmetry (scale ranges $0-0.5$ )

The results of the acoustic calculation for both cases with and without SAS are shown in Fig. 12 and Fig. 13. It can be seen a significant reduction in acoustic radiation in the case with SAS presence.

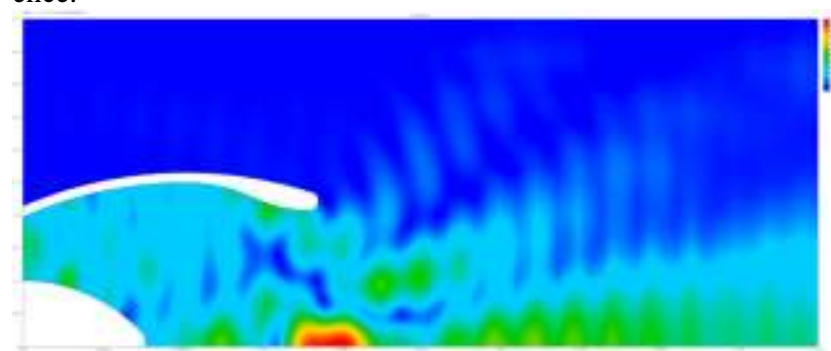




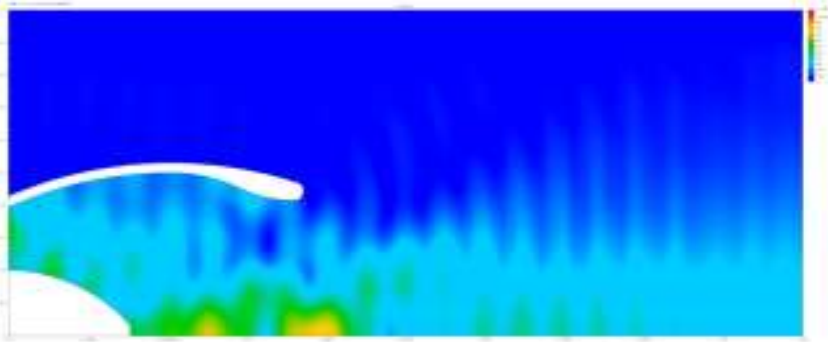

Fig. 12: Amplitude of pressure pulsations in the symmetry plane (scale range 0-1200 Pa). Top - without SAS, bottom - with SAS

Table 3 shows a total radiated acoustic energy and the effect of the SAS application. Reduction of emitted noise in terms of sound power comprises more than $6 \mathrm{~dB}$.

Table 3: Result of SAS application

\begin{tabular}{|l|l|l|}
\hline & Without SAS & With SAS Z=4-0.5i \\
\hline $\begin{array}{l}\text { Total radiated acoustic } \\
\text { energy }\end{array}$ & $24.1 \mathrm{~W}$ & $5.73 \mathrm{~W}$ \\
\hline Noise reduction & - & $6.25 \mathrm{~dB}$ \\
\hline
\end{tabular}

The calculations were performed on a processor i7 $3.2 \mathrm{GHz} \mathrm{CPU}$. Processor time for solving stationary problem takes around 16 minutes; the acoustic problem takes around 24 minutes. It ensures the applicability and effectiveness of this method in engineering practice where the most important requirement is a high performance: calculations of one case should take minutes of CPU time.

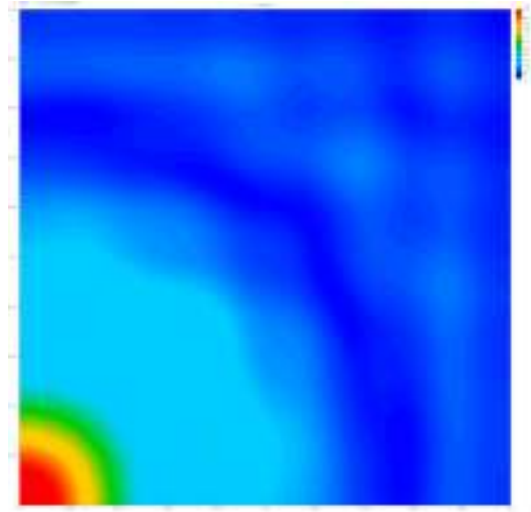

(a)

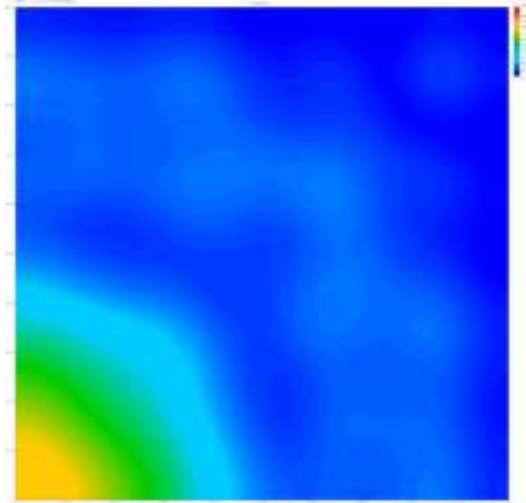

(b)

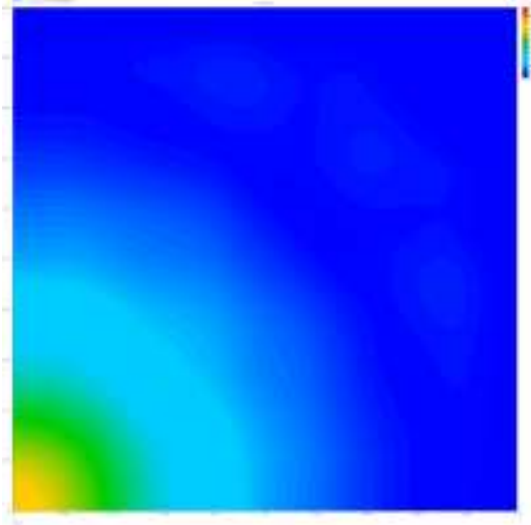

(c)

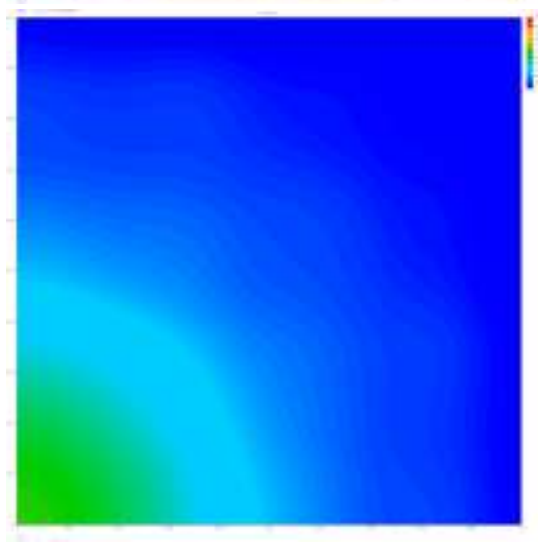

(d)

Fig. 13: Amplitude of pressure pulsations in the cross-sections before air inlet at $\mathrm{x}=0.7 \mathrm{~m}(\mathrm{a}, \mathrm{c})$ and $\mathrm{x}=1.1 \mathrm{~m}(\mathrm{~b}, \mathrm{~d})$ (scale range 0-600 Pa). (a, b) without SAS, (c, d) - with SAS

\section{Conclusion}

The proposed method of modeling the tonal component acoustic field is very efficient in terms of minimization of computer and human resources and calculation accuracy. It can be used for optimization of impedance characteristics and the SAS location for the turbofan engine BPF noise reduction.

\section{Acknowledgement}

This research was partly funded by Russian Foundation for Basic Research (RFBR) under application No. 17-58-560004.

\section{References}

[1] ISO 3740-1980 Acoustics - Determination of sound power levels of noise sources - Guidelines for the use of basic standards and for the preparation of noise test codes.

[2] ISO 11690-1 (1996), 'Acoustics - Recommended practice for the design of low-noise workplaces containing machinery - Part 1 : Noise-control strate-gies'.

[3] Jean Thimany Attacking Noise Early. Mechanical Engineering, No.8 (136), 2014, 44-49.

[4] Yan Jiang, Mats Åbom, Leping Feng, Sergey Timouchev, Christophe Maliczak Investigation of sound generation from an axial fan for engine cooling. Proceedings of 3rd International symposium on Fan Noise 2007, 19-21 September, 2007, Lyon, France.

[5] Yu. D. Khaletskii and A. S. Pochkin Fan noise reduction of an aircraft engine by inclining the outlet guide vanes. Acoustical Phys. 61(1), 2015, 101-108.

[6] A. Guédel Acoustique des ventilateurs. CETIAT. PYC LIVRES, 1999

[7] M.J. Lighthill 1952 Proceedings of the Royal Society, London A 211, 564-587. On sound generated aerodynamically. Part I. General Theory 
[8] N. Curle The influence of solid boundaries upon aerodynamic sound. Proc. Royal Soc. A 231, p.505-514, 1955

[9] J.E. Flowcs-Williams and D.L. Hawkings Philosophical Transactions of the Royal Society A264, 321-342. Sound generation by turbulence and surfaces in arbitrary motion , 1969

[10] W.R. Sears Some aspects of non-stationary airfoil theory and its practical application. Journal of the aeronautical sciences, vol. 8 (3), 1941

[11] H. Atassi, G. Hamad Sound generated in a cascade by threedimensional disturbances convected in a subsonic flow. Report NASA AIAA-81-2046, 1981

[12] J.M. Tyler, T.G. Sofrin Axial flow compressor noise studies. SAE Trans., Vol.70, 1962, pp. 309-332

[13] S. Caro, S. Moreau Comparaison d'une technique 2D de type Sears avec un calcul instationnaire direct pour le calcul du bruit de raie d'un ventilateur. Bruit des ventilateurs à basse vitesse. Actes du colloque tenu à l'Ecole Centrale de Lyon les 8 et 9 novembre 2001

[14] S. Caro, R. Sandboge, J. Iyer, Y. Nishio Presentation of a CAA formulation based on Lighthill's analogy for fan noise. Proceedings of 3rd International symposium on Fan Noise 2007, , Lyon, France, 19-21 September, 2007

[15] F. Farassat and M.K. Myers 1988 Journal of Sound and Vibration 123, 451-461. Extension of Kirchhhoff's formula to radiation from moving surfaces.

[16] A.A. Aksenov, V.N. Gavrilyuk and S.F. Timushev. Numerical Simulation of Tonal Fan Noise of Computers and Air Conditioning Systems. ISSN 1063_7710, Acoustical Physics, 2016, Vol. 62, No. 4, pp. 447-455. (C) Pleiades Publishing, Ltd., 2016. Original Russian Text (C) A.A. Aksenov, V.N. Gavrilyuk, S.F. Timushev, 2016, published in Akusticheskii Zhurnal, 2016, Vol. 62, No. 4, pp. 442-450.

[17] Timouchev S., Tourret J., Pavic G., Aksenov A. Numerical 2-D and 3-D methods for computation of internal unsteady pressure field and near-field noise of fans (Conference Paper) Noise Control Engineering Journal Volume 54, Issue 1, January 2006, Pages 15-20

[18] Синер А.А. Методика выбора звукопоглощающих конструкций для турбомашин на основе математического моделирования. Диссертация на соискание ученой степени кандидата технических наук, 2010

[19] M.K.Mayers. On the acoustic boundary condition in the presence of flow. - Journal of Sound and Vibration 71(3), September 1980 429-434.

[20] E. Redon, A.-S. Bonnet-Ben Dhia, J.-F. Mercier and S. Poernomo Sari. Non-reflecting boundary conditions for acoustic propagation in ducts with acoustic treatment and mean flow. International Journal for Numerical Methods in Engineering, 86, 2011, 1360-1378.

[21] A.A. Aksenov, V.N. Gavrilyuk and S.F. Timushev. Numerical Modeling of Fan Noise in Electronics. - NOVEM, 2015.

[22] A. A. Aksenov, A. A. Dyad'kin, V. A. Kutin, I. V. Moskalev, G. B Sushko, and S. A. Kharchenko, in Proc. All Russ. Sci. Conf. "Scientific Service in INTERNET Net; Solution of Large Problems" Novorossiisk, 2008.

[23] M. J. Aftosmis, M.J. Berger. Multilevel Error Estimation and Adaptive h-Refinement for Cartesian Meshes with Embedded Boundaries. AIAA 2002-0863. 40th AIAA Aerospace Sciences. Meeting and Exhibit. 14-17 January 2002 / Reno NVtesian Meshes with Embedded Boundaries.

[24] Mentor Graphics Corp. Advanced Immersed Boundary Cartesian Meshing Technology in FloEFD, 2011.

[25] Sushko G.B., Kharchenko S.A. Multi-threaded parallel implementation of the iterative algorithm for solving systems of linear equations with dynamic load balancing (in Russian). Proceedings of the international conference "Parallel computational technologies" (PACT'2008), Saint-Petersburg, 28 january - 1 february 2008. Cheljabinsk, JuUrGU publ., 2008, p.452-457.

[26] Sushko G.B., Kharchenko S.A. Experimental study on SKIF MGU "Chebyshev" of combined MPI+threads implementation of the iterative algorithm for solving SLAE's, appearing in FlowVision when modelling computational hydrodinamics problems (in Russian) Proceedings of the international conference "Parallel computational technologies" (PACT'2009), Nigniy Novgorod, 30 march -3 april 2009. Cheljabinsk, JuUrGU publ., 2009, p.316-324.

[27] Tester, B. J., "The propagation and attenuation of sound in lined ducts containing uniform or "plug" flow," Journal of Sound and Vibration 28(2), 1973, 151-203.

[28] A.A. Aksenov, V.N. Gavrilyuk and S.F. Timushev. Numerical Simulation of Tonal Fan Noise of Computers and Air Conditioning Systems. ISSN 1063_7710, Acoustical Physics, 2016, Vol. 62, No. 4, pp. 447-455. (C) Pleiades Publishing, Ltd., 2016. Original Russian
Text (C) A.A. Aksenov, V.N. Gavrilyuk, S.F. Timushev, 2016, published in Akusticheskii Zhurnal, 2016, Vol. 62, No. 4, pp. 442-450. 